\title{
Influence of Zika Virus in mechanisms related of cytotoxicity, cell adhesion, apoptosis and inflammatory markers on glioblastoma cells
}

Daniel Marinowic ( $\square$ daniel.marinowic@pucrs.br)

PUCRS https://orcid.org/0000-0002-1146-0745

Fabiana Spillari Viola

PUCRS

Fernanda Majolo

UNIVATES

Gabriele Goulart Zanirati

PUCRS

Pamella Nunes Azevedo

PUCRS

Ângela Zanatta

PUCRS

Ismael Plentz

PUCRS

Allan Marinho Alcará

PUCRS

Fernanda Bueno Morrone

PUCRS

Thamiris Becker Scheffel

PUCRS

Angélica Regina Cappellari

PUCRS

Paulo Michel Roehe

UFRGS

Ana Paula Muterle Varela

UFRGS

Denise Cantarelli Machado

PUCRS

Jaderson Costa Da Costa

PUCRS 


\section{Research Article}

Keywords: Zika virus, apoptosis, cell adhesion, glioblastoma, viral therapy

Posted Date: December 13th, 2021

DOI: https://doi.org/10.21203/rs.3.rs-1161905/v1

License: (c) (i) This work is licensed under a Creative Commons Attribution 4.0 International License. Read Full License 


\section{Abstract}

Glioblastoma (GBM) is one of the most common brain tumors in adults. Despite the presence of available treatments, it remains one of the most lethal and difficult tumors to treat such that most patients die within two years. Studies reported that infection with Zika virus (ZIKV) causes inhibition of cell proliferation as well as induction of apoptosis; moreover, these manifestations show a predilection for developing neuronal cells. In the present study, two GBM cell lines U-138 and U-251 were infected with ZIKV at multiplicities of infection (MOI) $0.1,0,01$ and 0.001 and tested for cell viability, cell migration, cell adhesion, induction of apoptosis, interleukin levels, and cell surface markers (CD14 and CD73). Our study demonstrated that the ZIKV infection promotes loss of cell viability and increased apoptosis potential. It was not evidenced changes in cell migration, however, the two glioblastoma cell lines displayed increased the cell adhesion behavior. There was small increase in the IL-4 level in the U-251 cell line after exposure to ZIKV, with no change in relation to INF-y levels. Furthermore, we observed an increase in the percentage of cells expressing the CD14 surface marker in both cell lines and increased CD73 expression in the U-251 cell line. Our results suggest that ZIKV may be associated with decrease of cell viability and increased CD73 expression, enhanced adherence, as well as increased apoptosis rates. Further investigations are required to explore the potential use of ZIKV in the treatment of GBM.

\section{Introduction}

Glioblastoma (GBM) is one of the most common primary and deadliest brain tumors in adults. Despite classical treatments consisting of surgery, radiotherapy, and chemotherapy, median survival is usually around 1 year (1). GBM is point as a tumor with extreme invasiveness potential and high recurrence rates. Moreover, cellular heterogeneity and difficulty in accessing the drugs by the blood-brain barrier make this disease difficult to treat (2). Based on the current knowledge of the complexity of this neoplasia, treatment strategies require a multidisciplinary approach, involving the pathophysiological understanding of invasion, migration, and resistance mechanisms (3).

Among the alternatives, viral therapy using Zika virus (ZIKV) has emerged as a novel strategy. Currently, there is evidence that ZIKV is capable of infecting and killing neural stem cells. Studies have shown the presence of viral DNA approximately $24 \mathrm{~h}$ after virus exposure in pluripotent stem cells, neural stem cells, neurospheres, and brain organoid cell cultures, with the most frequently encountered abnormalities being morphological and apoptotic induction (4). In embryonic brain tumor cell line and animal models of brain tumors, ZIKV infection-induced cell death and tumor regression (5).

Recently, Kaid et al. explored human cell lines to assess their susceptibility to ZIKV infection. Human neural progenitor cells were identified as a direct target of the virus, and as a consequence, it was observed significant reduction in the viable cell numbers, increased cell death, and dysregulation of the cell cycle (6). Also, a preclinical study with mouse-derived microglia cells that was subjected to ZIKV exposure demonstrated that the virus infected the microglia and was able to induce an inflammatory response. Consequently, these manifestations resulted in reduction of neurosphere size and growth, as 
well as inhibition of neuronal precursor cell proliferation. Together, these data demonstrate that microglia are highly susceptible to ZIKV infection that causes neuroinflammation and produces neurotoxic factors that are detrimental to the proliferation and differentiation of neuronal precursors (7).

Studies involving ZIKV-infected tumor stem cells have been shown that over $60 \%$ of tumor stem cells were infected with the virus within $48 \mathrm{~h}$ of viral exposure. Additionally, post-surgical resection isolated tumor stem cells were demonstrated to be infected when exposed to ZIKV, while normal neuronal cells were not affected. Markedly, using animal models of GBM, a reduction in tumor growth was observed, demonstrating that this viral agent, eventually modified, could serve as a potential model for viral therapy in GBM (8).

The features of GBM are implicated in its aggressivity. Migration process and invasiveness are the main cause of treatment problems, mostly involved in tumor recurrence. CD73 is an important ectoenzyme of the purinergic system, present in the extracellular space and the main source of adenosine (9). CD73 has been found to be overexpressed in several types of cancers, including GBM, and its role as a membrane protein assisting cell adhesion and migration processes was described (10). Additionally, CD14 is a surface marker expressed preferentially in the cells of the immune system. In certain cancers, CD14 expression is required for increased cytokine production and increased tumor growth. Tumors formed by CD14 ${ }^{\text {high }}$ cells showed to be very vascularized, presenting greater myeloid cell infiltration rates (11).

The neurotoxic effects observed in cases of microcephaly along with recent data suggesting the ZIKVtropism for immature neuronal cells could become the ZIKV-based therapy a promising strategy against GBM growth. In the present study, we aim to explore the effect of Zika virus infection on cell viability and the migration potential of human GBM cell lines.

\section{Results}

\section{ZIKV infection promotes loss of cell viability and apoptosis in GBM cells}

To assess the effect of ZIKV infection on GBM cells viability, we performed the MTT assay testing different $\mathrm{MOI}$ concentrations $(0.1,0.01$, or 0.001$)$ at $2 \mathrm{~h}$ of exposure. Interestingly, GBM cells showed a greater sensibility to ZIKV infection. The virus was able to reduce around $60 \%$ the viability of U-138 cells at $\mathrm{MOI} 0.001$ (Figure 1A). The data suggest that the virus is causing damage to tumor cells while not appearing to affect normal cells. It was observed that the virus cannot induce large effects on the viability of Vero and U-251 cells. Despite the viability remaining near $100 \%$ in all tested $\mathrm{MOI}$, a small fluctuation in cell viability was detected after $2 \mathrm{~h}$ of exposure (Figure 1B and 1C). The reduction of GBM viability seems to be related to apoptosis at the trigger according to the data presented in (Figure 1D). The triplex assay showed no change in the cell viability and citotoxicity at either time ( 2 and $12 \mathrm{~h}$ ), and either $\mathrm{MOI}(0.1$ and 0.01 in GBM cell lines) In the Vero control cell line there was a reduction in citotoxity in the $12 \mathrm{~h}$ time. Interestingly, there was an increase in the apoptosis rate in U-251 and U-138 cell lines when exposed for 2 
$\mathrm{h}$ to $\mathrm{MOI} 0.1$ of ZIKV as compared to uninfected cells. At the same dilution, after $12 \mathrm{~h}$ of exposure, the apoptosis rate of U-251 cell line was similar to that of non-ZIKV infected cells. The U-138 cell line displayed an increased apoptosis rate at $12 \mathrm{~h}$ exposure compared to uninfected cells. The exposure to $\mathrm{MOI} 0.01$ at 2 and $12 \mathrm{~h}$ showed similar values of apoptosis rates in this cell line (U-138). At the same dilution, $\mathrm{U}-251$ cell line showed a decrease in apoptosis rate over $12 \mathrm{~h}$.

\section{Effects of ZIKV exposure in cell migration and adhesion}

Cell migration assay showed no statistically significant difference in the distance between the regions where cells migrated at any of the ZIKV dilutions within 2 and 24-hour exposure period compared to uninfected (control) cells. The cells showed similar migratory behavior when compared to $10 \%$ serumsupplemented cultures. None of the concentrations tested could alter the cell migration potential of the analyzed cell lines (Figure 2A). ZIKV exposure increased the adhesion potential of U-138 and U-251 GBM cell lines (Figure 2B). Vero cell line did not change its cell adhesion potential after exposure to two different MOI. For GBM cell lines, $2 \mathrm{~h}$ of ZIKV exposure increased the cell adhesion rate. There was no statistically significant difference between the groups analyzed. The U-138 cell line showed a $32 \%$ increase in the cell adhesion potential at $\mathrm{MOI} 0.1$, and $42 \%$ at $\mathrm{MOI} 0.01$, when compared with the same cell line without virus contact. In U-251 cell line, the increase in adhesion potential was $39 \%$ at $\mathrm{MOI} 0.1$ and $40 \%$ at $\mathrm{MOI} 0.01$ when compared with the same uninfected cell line (Figure 2B).

\section{ZIKV infection increases CD14 ${ }^{+}$an $\mathrm{CD}^{+} 3^{+}$markers and enhances the expression of inflammatory-related markers}

To understand the immune signaling triggered by the ZIKV, inflammatory markers were investigated (Figure 3). We showed that GBM cells reacted to ZIKV infection presenting a tendency to greater expression of CD14 when compared to normal cells (Vero) (Figure 3A). Two GBM cell lines were used to explore the immune response against ZIKV, once there are distinct features among lineages. Both U-138 and U-251 presented a similar response after $2 \mathrm{~h}$ after exposure to ZIKV. The percentage of U-138 cells labeled with CD14 was $1.78 \%$. After $2 \mathrm{~h}$ of exposure to ZIKV, this percentage changed to $2.82 \%$ at $\mathrm{MOI}$ 0.01 . For the U-251 cells, the exposure to the virus doubled the percentage of CD14 ${ }^{+}$, changing from $1.8 \%$ for uninfected cells to $3.55 \%$ when exposed to $\mathrm{MOI} 0.001$ (Figure 3A). There was a statistically significant increase in CD73 expression in U-251 cell line at three different dilutions analyzed. The percentage of labeled cells in the uninfected cell line was $14.41 \%$, increasing to $25.8 \%$ after exposure to ZIKV at MOI 0.1 and $29.3 \%$ at $\mathrm{MOI} 0.01$. In U-138 cell line, there was no significant increase in the percentage of CD73labeled cells after exposure to different ZIKV concentrations (Figure 3B).

The expression of surface marker CD14 has been inversely correlated to the expression of inflammatory cytokines (12). In order to understand the mechanisms involved in the effects exerted by virus treatment, inflammatory markers were investigated. ZIKV reduced the levels of INF- $Y$ and IL-4 in the Vero cell line after $2 \mathrm{~h}$ of exposure compared to the unexposed control (Figure $3 \mathrm{C}$ and $\mathrm{D}$ ). The exposure of ZIKV to U- 
251 and U-138 cell line appeared not changed the levels of the INF- $y$ and IL-4after $2 \mathrm{~h}$ of exposure as compared to its unexposed control.

\section{Discussion}

In the present study, human GBM cell lines U-251 and U-138 were used to evaluate the effects of ZIKV infection under different dilutions and exposure times. According to our results, after $2 \mathrm{~h}$ of exposure to ZIKV (MOI 0.1) we observed a reduction in cell viability in the U-251 human GBM cell line. According to our results, no changes were identified in cellular viability for the three cell lines studied after exposure to ZIKV in Triplex assay. In the same assay, we analyzing this same parameter using the Triplex assay, no changes were identified for the three cell lines studied after exposure to the virus. Current in vitro viral replication studies provide detailed information on infection-related virological variables, such as the amount of virus and number of target cells measured over time, and it is extremely difficult to translate these data quantitatively through parameters that identify the extremely variable kinetics of viral infection. Mathematical value models based on different virus parameters are currently available to identify the main functional differences between viruses and to understand differences related to their virulence and different time course and the number of harmful post-infection viral particles (13).

We observed an increase in caspases 3 and 7 rate in both cell lines exposed to MOI 0.1 of ZIKV, as compared to the uninfected cells after $2 \mathrm{~h}$ of viral exposure. At the same dilution, after $12 \mathrm{~h}$ of viral exposure, there was no difference in the U-251 cell line compared to the control, and in U-138, there was an increase in the apoptosis rate after $12 \mathrm{~h}$ of viral exposure. These results may lead to the hypothesis that ZIKV-mediated apoptosis induction may start in the early stages of viral infection, lasting for longer periods and that this effect also occurs at lower dilutions and does not appear to be dependent on higher viral concentrations. We can also suggest that the genetic and phenotypic variations among the studied cell lines describe different profiles of response to ZIKV infection, which may be altering different cell pathways and inducing apoptosis cell death. This is corroborated by the finding that a significant increase in apoptosis was observed in the $\mathrm{U}-251$ cell line after $2 \mathrm{~h}$ of exposure, only in the most concentrated form of the virus ( $\mathrm{MOI} 0.1$ ). For $\mathrm{U}-138$ cell line, this effect was more prominent, being observed after 2 and $12 \mathrm{~h}$ of exposure at MOI 0.1. ZIKV is known to have a predilection for undifferentiated cells and pluripotent neuronal cells, including tumor cells of the same tissue. Kaid et al. (2018) exposed three different brain tumor cell lines to ZIKV and similarly obtained different effectiveness of the virus in reducing cell numbers. Moreover, they also demonstrated a predilection of ZIKV for tumor cells over neural progenitor cells, indicating that the virus has a selectivity to cancer cells and promote cell death at different intensities (5).

In addition to the intense cell proliferation characteristic of GBM, its highly invasive and infiltrative profile makes it one of the most difficult to treat malignancies. Besides, this tumor is characterized by cell heterogeneity, maintenance of tumor stem cells, genetic alterations such as mutations, deletions, and epigenetic changes that interfere with different intracellular signaling processes. Furthermore, host 
immune alterations, leading to the reasoning that for improved treatment, we should consider the mechanisms that may be more related to the maintenance of the malignant phenotype (2).

In this context, we evaluated the ability of ZIKV to modulate invasive processes in human GBM cell lines. In the wound healing assay that refers to a block or cell cluster migration model, we did not observe any significant change after exposure to the virus, which was different from the results described in the literature that ZIKV reduced the migratory capacity of developing neuronal cells. However, this effect on tumor cells is not yet known (7). We observed that in U-138 cells at the three times analyzed and in the $\mathrm{MOI} 0.01$, there was an increase in cell adhesion, evident only in tumor lineages. Adhesion and migration events together constitute the cell motility processes. Sequential events of integrin binding to extracellular matrix proteins associated with cytoskeleton protrusion favor individual cell migration, constituting a characteristic movement of mesenchymal and tumor cell phenotypes such as GBMs (14). When there is an increase in the adhesion capacity, these cells cannot detach from the substrate quickly, thereby reducing cell migration, as observed. Adhesion between cells is a process that normally occurs in healthy cells. In tumors, usually the proteins that make this cell adhesion have altered expression, resulting in reduced adhesion between the tumor cells, thus allowing their detachment from the surrounding tissues. In GBM, this is an extremely interesting finding, as this disease has a great ability to infiltrate adjacent brain areas, making it difficult to control through surgery and radiotherapy. Thus, the ability to keep the cells adhered would hinder the process of local invasion characteristic of the disease. The literature shows that ZIKV treatment could reduce spinal cord metastasis levels by $60 \%$ after brain tumor implantation in orthotopic implant xenographic models (5). Therefore, we can suggest that ZIKV exposure reduces GBM cell invasiveness by inducing an increase in cell adhesion.

In addition to integrins, numerous other molecules participate in the cell adhesion process (14). Earlier studies have shown that CD73 protein is a regulatory molecule involved in cell proliferation, migration, and invasion $(10,15)$. Moreover, in vitro studies have shown that the dysregulation of its activity can cause reduced adhesion in the GBM cell line (16). Therefore, the increase of CD73 expression in U-251 cell line may corroborate the increase in adhesion potential after exposure to different ZIKV concentrations. CD73 is widely expressed in cells with a high degree of undifferentiation; it is currently one of the proteins used as markers of mesenchymal cells (17). Different types of tumors, such as breast cancer, melanoma, GBMs, and multiform GBMs, have high CD73 expression and activity. Its association with the intense proliferative and invasive capacity of these tumors allows this membrane protein to act as a marker of a high degree of tumor malignancy (18). Considering that ZIKV has a high affinity for undifferentiated cells of neural origin and also for tumor cells of the same tissue and that CD73 is a membrane protein widely expressed in these two cell types, we can suggest that the modulation of ZIKV expression of this protein in GBM cell lines may characterize an important mechanism of action, which modulates the invasiveness of GBM cells via increased CD73 expression.

The increased expression of CD14 in the studied cell lines is probably a consequence of stimulation of the immune system, which may represent important information owing to the fact that glioblastoma is considered a non-reactive tumor in relation to the immune system, a fact that has hindered the 
acquisition of good results with immunotherapy, which has been very promising in other tumors (11). The fact that it stimulates the immune system in the tumor microenvironment is an interesting information, as the combination of treatments involving oncolytic viruses with immunotherapy has been studied, which seems to be an opportunity to investigate the impact of combining immunostimulatory agents as oncolytic viruses with immunotherapy strategies, effecting the anti-tumor immune response (19).

Exposure for $2 \mathrm{~h}$ to ZIKV increased the amount of INF-Y in the U-251 cell line at MOI 0.1 when compared to uninfected controls, gradually decreasing with increasing virus dilution, but not in U-138 cell line that displayed similar levels of INF- $\gamma$ when compared to the control. Ari Kane et al. have shown that increased INF-y disrupts GBM proliferation, induces apoptosis, increases tumor immunogenicity, and inhibits neovascularization. This increase in INF-y mediated by ZIKV action at MOI 0.1 in U-251 cell line could be translated as an adjuvant tool for treating GBM as it is a cytokine that acts on cell surface receptors, thereby activating the transcription of genes that increase tumor immunogenicity, interrupt proliferative mechanisms, and inhibit tumor angiogenesis (15).

The U-251 cell line also showed an increase in IL-4 levels at MOI 0.1 and a reduction even at levels lower than the uninfected control at MOI 0.01 after $2 \mathrm{~h}$ of ZIKV exposure. IL-4 plays a critical role in regulating immune responses and has been detected at high levels in the tumor microenvironment of cancer patients, where concentrations of this interleukin correlate with the degree of malignancy (20).

Owing to the complexity associated with the tumor microenvironment, GBM is characterized by low efficacy of currently available treatments, including the absence of several tumor molecular targets already studied, as well as the lack of significant response to immunotherapy. Understanding of the functioning of the tumor microenvironment, gene expression, and molecular alterations found in the GBM pathology is crucial for exploring new treatment modalities. The current knowledge regarding the use of oncolytic viruses would help us to explore this form of treatment, used alone or in combination with chemotherapy or immunotherapy agents.

ZIKV has very interesting features that potentially justify its use for treating brain tumors such as GBM such as apoptosis induction, reduction of neuronal and developing cell migration capacity, and potential induction of the host immune system. The results of our study confirm some of these effects and indicate novel mechanisms involved in the interaction of ZIKV with GBM tumor cells. Further studies need to be conducted using animal models to present and define gaps such as dose to be used, route of exposure to the viral agent, genetic modification of ZIKV to reduce the systemic effects of viral infection. This would translate the ZIKV methodology into a more feasible and safe approach for treating brain tumors.

The exposure of U-251 and U-138 glioblastoma cell lines to different dilutions and time of ZIKV did not lead to changes in cell viability and migration changes; however, there was an increase in apoptosis and adhesion potential of both GBM cell lines. Moreover, there was a reduction in IL-4 expression in the U-251 cell line after exposure to ZIKV for $2 \mathrm{~h}$, with no change in relation to INF-y levels. Finally, there was an increase in the percentage of cells expressing the CD14 surface marker in U-138 and U-251 cell lines and 
an increase in CD73 expression in the U-251 cell line. Corroborating the results found in the literature, we present here non-lethal mechanisms of action of ZIKV on U-251 and U-138 glioblastoma cell lines. The set of alterations observed such as increased expression of CD73, enhanced the potential for cell adhesion, not influencing migration but reducing its invasive capacity, and increased apoptosis, can keep the tumor static, reducing its invasive capacity in loco, and being a more susceptible target to chemotherapy, radiotherapy, or surgery. Following the several necessary advances related to the safety and feasibility of using ZIKV therapy for GBM, we could suggest that this therapy should be explored as a potential agent in the current treatment of this tumor.

\section{Material And Methods}

\section{Cell lines}

Human GBM cell lines U-138 and U-251 were obtained from ATCC (Rockville, MD, USA). Vero cell line (African green monkey kidney epithelial cells) was used as the non-malignant control available at the Brain Institute (Bralns) and at the Applied Pharmacology Laboratory (PUCRS). These cell lines were grown in Dulbecco's modified Eagle medium (DMEM) supplemented with $10 \%$ bovine fetal serum, 100 $\mathrm{U} / \mathrm{ml}$ penicillin, $100 \mathrm{U} / \mathrm{ml}$ streptomycin, $100 \mu \mathrm{g} / \mathrm{ml}$ gentamicin under $5 \% \mathrm{CO}_{2}$ at $37^{\circ} \mathrm{C}$. For exposure to ZIKV, cell lines were cultivated in culture plates specific to each type of analysis methodology.

\section{Zika virus infection}

Brazilian ZIKV strains 17 (ZIKV 17) was isolated from a 33-year-old patient with clinical features suggestive of ZIKV infection in 2016. Virus was propagated in Vero E6 cell line (ATCC CRL-1586), titrated using a standard plaque assay $(13,21)$ and viral stocks were maintained at $-80^{\circ} \mathrm{C}$. Human GBM cells U138 and U-251 were infected with ZIKV 17 at multiplicity of infection (MOI) 0.1, 0.01 and 0.001.

\section{Cell viability assay}

The 96 -well plates were seeded with $4.5 \times 10^{5}$ cells per well containing $200 \mu \mathrm{l}$ of supplemented DMEM medium. The cells were cultured for $24 \mathrm{~h}$ and after this period were infected with ZIKV at different $\mathrm{MOI}$ for $2 \mathrm{~h}$. The cells were washed with Dulbecco's phosphate-buffered saline (DPBS), followed by the addition of $200 \mu \mathrm{l}$ of (3-(4,5-dimethylthiazol-2-yl)-2,5-diphenyltetrazolium bromide) MTT solution at a concentration of $5 \mathrm{mg} / \mathrm{ml}$ in supplemented DMEM medium. Cells were incubated for $4 \mathrm{~h}$ at $37^{\circ} \mathrm{C}$ in a $5 \%$ $\mathrm{CO}_{2}$ incubator. MTT was solubilized in $200 \mu$ of dimethyl sulfoxide (DMSO). Optical densities were measured at a wavelength of $570 \mathrm{~nm}$ in an ELISA reader. The absorbance values attributed to uninfected cells served as beacons for calculating the cell viability percentage.

\section{Triplex assay}

After prior ZIKV exposure of GBM and control Vero cell lines for $2 \mathrm{~h}$ and $12 \mathrm{~h}$ at different MOI dilutions, the cells were trypsinized and cultured in a 96-well plate at a density of $2 \times 10^{4}$ cells per well in triplicate. For quantification of viability/cytotoxicity with GF-AFC and bis-AAF-R110 substrates, $20 \mu$ l of the solution 
was added and the cells were placed under orbital shaking at $300 \mathrm{rpm}$ for $30 \mathrm{~s}$, followed by incubation at $37^{\circ} \mathrm{C}$ for $30 \mathrm{~min}$. To measure cell viability and cytotoxicity, the first reading was taken at wavelengths of $400 \mathrm{~nm}$ for excitation and $505 \mathrm{~nm}$ for emission for viability, and $485 \mathrm{~nm}$ for excitation and $520 \mathrm{~nm}$ for emission for cytotoxicity. After reading, Caspase-Glo® 3/7 reagent was added, and the plate was incubated again at $37^{\circ} \mathrm{C}$ for $30 \mathrm{~min}$. The reading to measure caspase $3 / 7$ activity was taken in the luminescence mode. All readings were taken using the SpectraMax M plate reader.

\section{Cell migration assay}

Evaluation of cell migration was performed as described by Valster et al. (22). The GBM cell lines, U-251 and U-138, and Vero cells were seeded in 12-well plates and cultured until confluent. Sequentially, the cells were submitted to the progressive reduction of FBS concentration until $0.5 \%$ and exposed to ZIKV for $2 \mathrm{~h}$. Next, cells were washed with DPBS and two parallel scratch wounds were created on the monolayer with a P200 pipet tip. DPBS was removed and cells were again cultured in the DMEM medium supplemented with $0.5 \%$ FBS. Images were captured at 0,12 , and $24 \mathrm{~h}$. The images were analyzed using ImageProPlus7 software and the cell migration distance was determined by measuring the width of the wound divided by two and by subtracting this value from the initial half-width of the wound.

\section{Cell adhesion assay}

After exposure to ZIKV for $2 \mathrm{~h}$ at two different $\mathrm{MOI}$, the cell lines were trypsinized and grown in 96-well triplicate plates at $10^{4}$ cell density per well in $100 \mu$ I DEM supplemented with $10 \%$ FBS. After $2 \mathrm{~h}$ of incubation, the culture medium was aspirated, and the cells were washed with DPBS and fixed with $4 \%$ paraformaldehyde (PFA) for $10 \mathrm{~min}$. The cells were again washed with DPBS, following which $50 \mu \mathrm{l}$ of $0.5 \%$ violet crystal dissolved in $20 \%$ methanol was added per well. After $10 \mathrm{~min}$, the dye was aspirated and $50 \mu \mathrm{l}$ of acetic acid was added per well. The measurement was performed using a plate reader at a wavelength of $570 \mathrm{~nm}$.

\section{Luminex immunoassay}

The Luminex immunoassay was performed to measure the levels of INF-y and IL-4 in the culture supernatant of cell lines after exposure to ZIKV for $2 \mathrm{~h}$ at different dilutions. Magnetic beads corresponding to each interleukin were added to the 96 -well plate. Next, $50 \mu$ of each sample and each dilution point of the standard curve were added. The plate was subsequently shaken at $500 \mathrm{rpm}$ for $2 \mathrm{~h}$ and washed using wash buffer and the detection antibody was added. The plate was shaken at $500 \mathrm{rpm}$ for $30 \mathrm{~min}$, followed by addition of $50 \mu \mathrm{l}$ streptavidin. The plate was washed using wash buffer and read on Luminex MAGPIx equipment.

\section{Flow cytometry}

After exposure to ZIKV, the cells were incubated for $2 \mathrm{~h}$ with anti-CD14 antibody (Thermo Scientific) conjugated to PE and anti-CD73 antibody (Thermo Scientific) conjugated to FITC antibodies. After incubation, the cells were washed with DBPS and the labeled cell population was estimated for each antibody by flow cytometry using the BD FACSCalibur (BD Bioscience) equipment. 


\section{Statistical analysis}

The cell viability, cell migration, cell adhesion, and flow cytometry assays were repeated at two different times in triplicate $(n=3)$. The values were considered statistically significant when $p<0.05$. For triplex and cell viability assays, analysis of variance was performed using the two-way ANOVA test with Bonferroni's post-test. Cell adhesion and Luminex assays, analysis of variance was performed by oneway ANOVA with Tukey's post-test.

\section{Ethical aspects}

The present work was approved by the Scientific Committee of the Brain Institute of PUCRS under number 8249 and by the National System of Management of Genetic Heritage and Associated Traditional Knowledge (SISGEN) under accession number A708680.

\section{Declarations}

\section{Funding}

This study was financed in part by the Coordenação de Aperfeiçoamento de Pessoal de Nível Superior Brazil (CAPES) - Finance Code 001, Conselho Nacional de Desenvolvimento Científico e Tecnológico (CNPq) and Pandurata Alimentos Ltda. J.C. Da Costa is CNPq researcher.

\section{Conflict of Interest}

The authors declare that they have no competing interests.

\section{References}

1. Stupp R, Mason WP, J van den BM, Weller M, Fischer B, Taphoorn MJB, et al. Radiotherapy plus Concomitant and Adjuvant Temozolomide for Glioblastoma. N Engl J Med. 2005;352:987-96.

2. Cai $X$, Sughrue M. Glioblastoma: new therapeutic strategies to address cellular and genomic complexity. Oncotarget. 2018;9:9540-54.

3. Dunwoodie L, Poehlman W, Ficklin S, Feltus F. Discovery and validation of a glioblastoma coexpressed gene module. Oncotarget. 2018;9:10995-1008.

4. Tang H, Hammack C, Ogden SC, Wen Z, Qian X. Zika Virus Infects Human Cortical Neural Precursors Attenuates Growth 2016 Tang. 2017;18(5):587-90.

5. Kaid C, Goulart E, Caires-Júnior LC, Araujo BHS, Soares-Schanoski A, Bueno HMS, et al. Zika virus selectively kills aggressive human embryonal CNS tumor cells in vitro and in vivo. Cancer Res. 
2018;78(12):3363-74.

6. Gabriel E, Ramani A, Karow U, Gottardo M, Natarajan K, Gooi L, et al. Recent Zika Virus Isolates Induce Premature Differentiation of Neural Progenitors in Human Brain Organoids. Cell Stem Cell. 2017;20:397-406.

7. Wang J, Liu J, Zhou R, Ding X, Zhang Q, Zhang C, et al. Zika virus infected primary microglia impairs NPCs proliferation and differentiation. Biochem Biophys Res Commun [Internet]. 2018;497(2):61925. Available from: https://doi.org/10.1016/j.bbrc.2018.02.118

8. Zhu Z, Gorman MJ, McKenzie LD, Chai JN, Hubert CG, Prager BC, et al. Zika virus has oncolytic activity against glioblastoma stem cells. J Exp Med. 2017;214(10):2843-57.

9. Yegutkin GG. Enzymes involved in metabolism of extracellular nucleotides and nucleosides: Functional implications and measurement of activities. Crit Rev Biochem Mol Biol. 2014;49(6):47397.

10. Azambuja J, Gelsiecichter N, Beckenkamp L, Iser I, Fernandes M, Figueiró F, et al. CD73 Downregulation Decreases In Vitro and In Vivo Glioblastoma Growth. Mol Neurobiol. 2019;56:32602379.

11. Cheah M, Chen J, Sahoo D, Contreras-Trujillo H, Volkmer A, Scheeren F, et al. CD14-expressing cancer cells establish the inflammatory and proliferative tumor microenvironment in bladder cancer. Proc Natl Acad Sci U S A. 2015;112:4725-30.

12. Rey Nores JE, Bensussan A, Vita N, Stelter F, Arias MA, Jones M, et al. Soluble CD14 acts as a negative regulator of human T cell activation and function. Eur J Immunol. 1999;29(1):265-76.

13. Iwami S, Sato K, De Boer RJ, Aihara K, Miura T, Koyanagi Y. Identifying viral parameters from in vitro cell cultures. Front Microbiol. 2012;3(SEP):1-6.

14. Friedl P, Wolf K. Plasticity of cell migration: a multiscale tuning model. J Cell Biol. 2010;188:11-9.

15. Kane A, Yang I. Interferon-gamma in Brain Tumor Immunotherapy. Neurosurg Clin N Am [Internet]. 2010;21(1):77-86. Available from: http://dx.doi.org/10.1016/j.nec.2009.08.011

16. Cappellari A, Vasques G, Bavaresco L, Braganhol E, Battastini A. Involvement of ecto-5'nucleotidase/CD73 in U138MG glioma cell adhesion. Mol Cell Biochem. 2012;359:315-22.

17. Calloni R, Cordero E, Henriques J, Bonatto D. Reviewing and updating the major molecular markers for stem cells. Stem Cells Dev. 2013;22:1455-76.

18. Soleimani A, Taghizadeh E, Shahsavari S, Amini Y, Rashidpour H, Azadian E, et al. CD73; a key ectonucleotidase in the development of breast cancer: Recent advances and perspectives. $\mathrm{J}$ Cell Physiol. 2019;234(9):14622-32.

19. Lawler S, Speranza M, Cho C, Chiocca E. Oncolytic Viruses in Cancer Treatment: A Review. JAMA Oncol. 2017;3:841-9.

20. Nappo G, Handle F, Santer FR, McNeill R V., Seed RI, Collins AT, et al. The immunosuppressive cytokine interleukin-4 increases the clonogenic potential of prostate stem-like cells by activation of 
STAT6 signalling. Oncogenesis [Internet]. 2017;6(5):e342-12. Available from:

http://dx.doi.org/10.1038/oncsis.2017.23

21. Fontes-Garfias C, Shan C, Luo H, Muruato A, Medeiros D, Mays E, et al. Functional Analysis of Glycosylation of Zika Virus Envelope Protein. Cell Rep. 2017;21:1180-90.

22. Valster A, Tran NL, Nakada M, Berens ME, Chan AY, Symons M. Cell migration and invasion assays. Methods. 2005;37(2):208-15.

\section{Figures}

\section{Figure 1}

Representation of cell toxicity analysis after $2 \mathrm{~h}$ of cell exposure at different ZIKV MOI (0.1, 0.01 and 0.001). Cell toxicity was analyzed by MTT assay and the values are represented by the mean and standard deviation of the percentage of viable cells compared to cells not exposed to the virus in U-138 (A), U-251 (B) and Vero (C). The Figure 1D show the cell viability, cytotoxicity and apoptosis analysis by U251, U-138 and Vero cell line by Triplex assay prior to the exposure to ZIKV for 2 and $12 \mathrm{~h}$ at virus. The values are based on the fluorescence for viability and cytotoxicity and luminescence for apoptosis.

A.
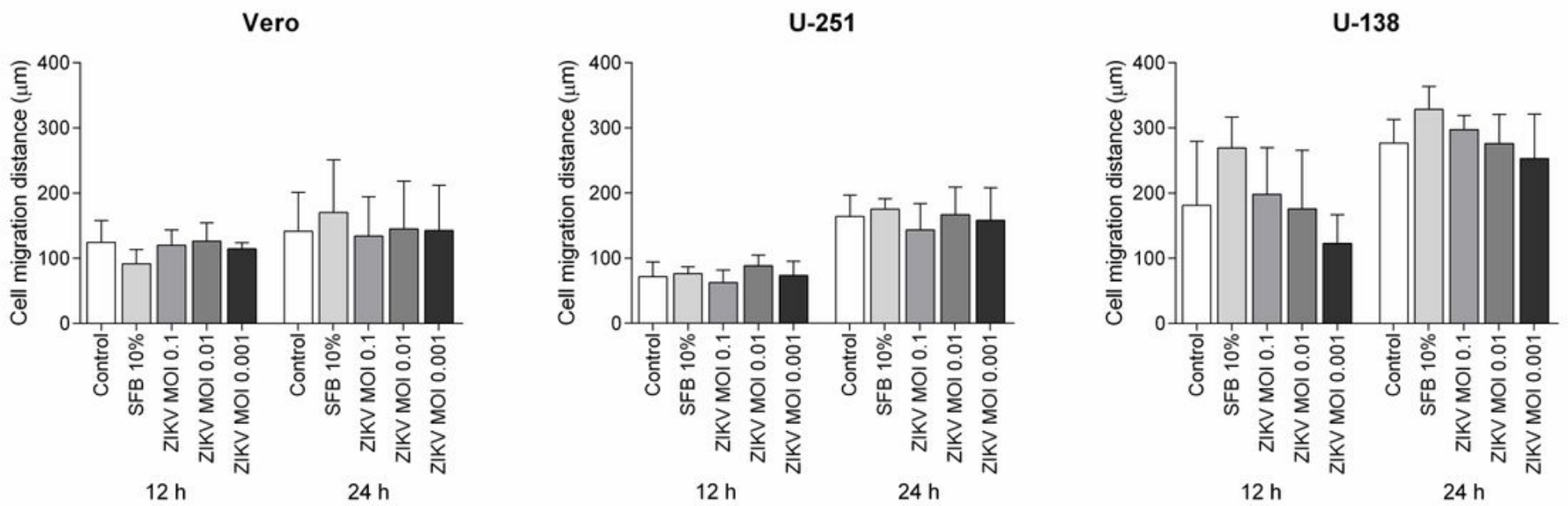

B.

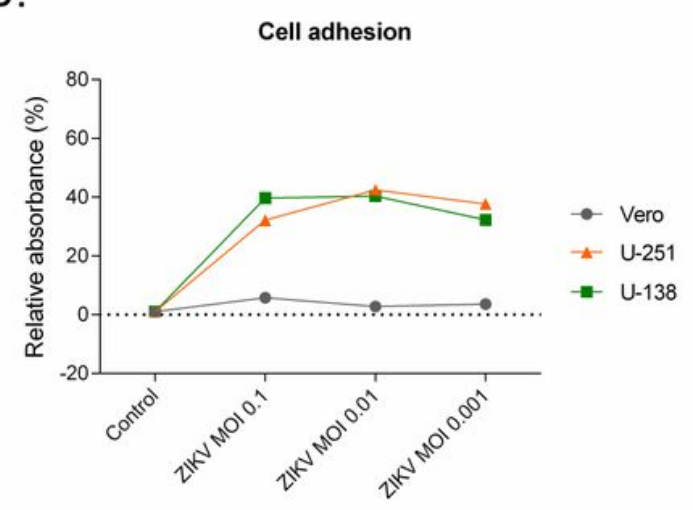


Figure 2

(A) Cell migration assay by measuring the distance between the two areas containing cells. The images were taken 12 and $24 \mathrm{~h}$ after the beginning of the analysis. The measurements are presented in micrometers $(\mu \mathrm{m})$ of the distance between the cell-containing ends and represent the capacity of cell migration after exposure to each dilution of ZIKV. (B) Vero, U-138, and U-251 cell lines adhesion assay on uninfected cells (Control) and infected cells at different ZIKV dilutions. The values are represented by the absorbance of the cells adhered to the plates and stained with $0.5 \%$ violet crystal.

A

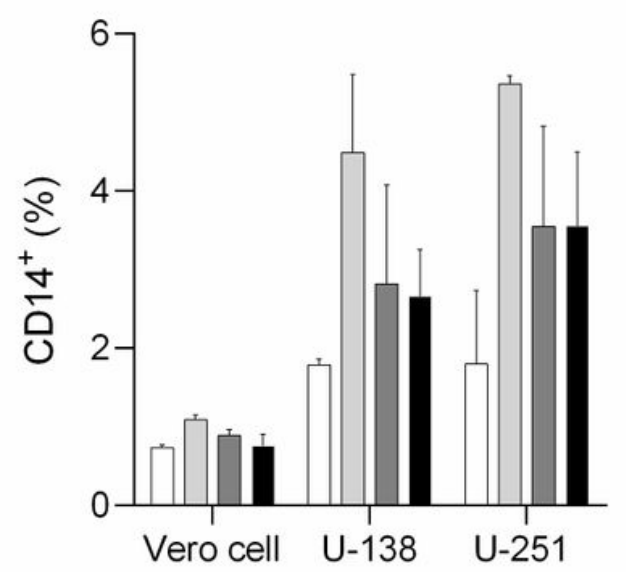

C

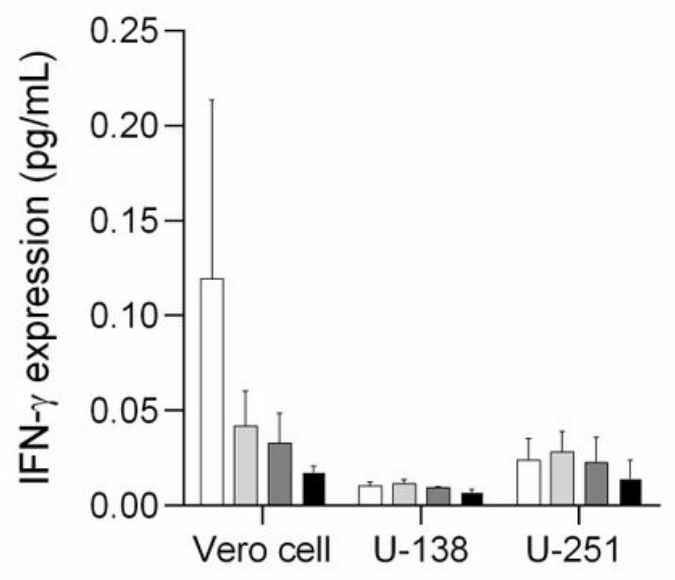

B

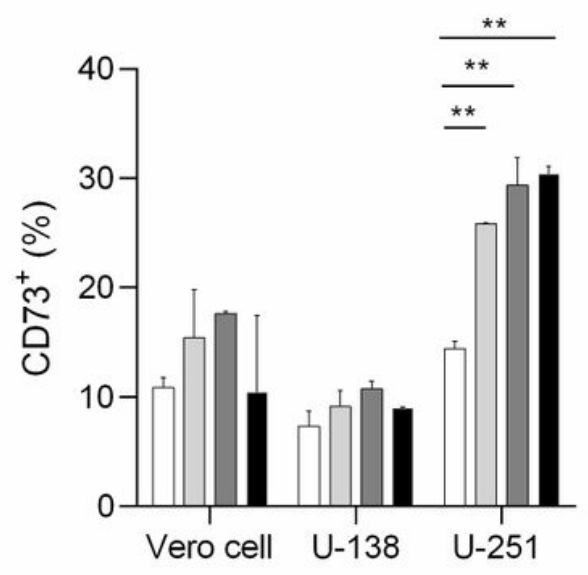

D

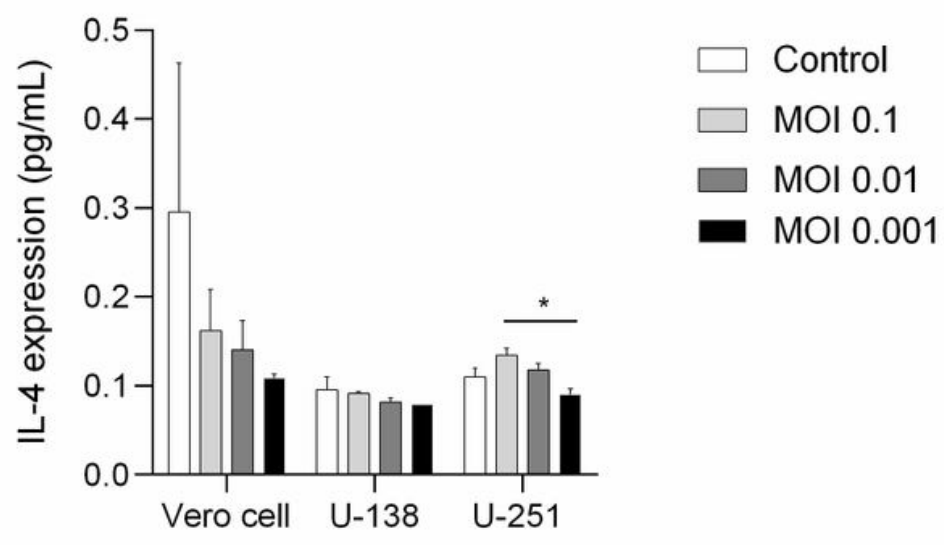

Figure 3

Expression profile representing CD14 (A) and CD73 (B) markers and determined by plotting the mean percentage of positive cells for each surface marker after exposure to different ZIKV MOI. Immunoassay for interleukins INF-Y (C) and IL-4 (D) represented by absolute quantification in picograms per milliliters $(\mathrm{pg} / \mathrm{mL})$ by comparison with the standard curve. The results are presented as mean \pm standard deviation. 